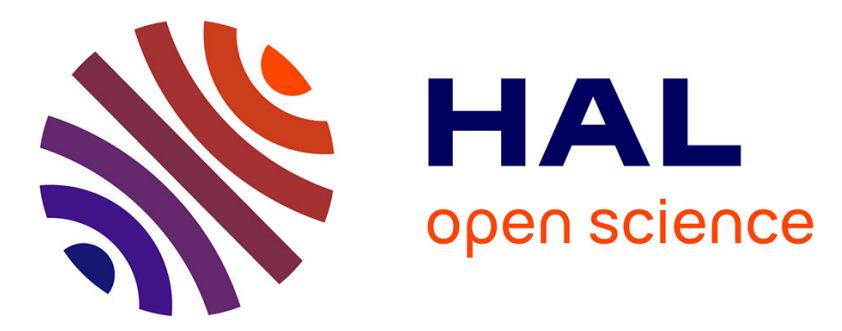

\title{
TRADR Project: Long-Term Human-Robot Teaming for Robot Assisted Disaster Response
}

Ivana Kruijff-Korbayová, Francis Colas, Koen Hindriks, Mark Neerincx, Petter Ögren, Mario Gianni, Tomáš Svoboda, Rainer Worst

\section{To cite this version:}

Ivana Kruijff-Korbayová, Francis Colas, Koen Hindriks, Mark Neerincx, Petter Ögren, et al.. TRADR Project: Long-Term Human-Robot Teaming for Robot Assisted Disaster Response. KI - Künstliche Intelligenz, 2015, 29 (2), pp.193-201. 10.1007/s13218-015-0352-5 . hal-01143484

\author{
HAL Id: hal-01143484 \\ https://hal.science/hal-01143484
}

Submitted on 17 Apr 2015

HAL is a multi-disciplinary open access archive for the deposit and dissemination of scientific research documents, whether they are published or not. The documents may come from teaching and research institutions in France or abroad, or from public or private research centers.
L'archive ouverte pluridisciplinaire HAL, est destinée au dépôt et à la diffusion de documents scientifiques de niveau recherche, publiés ou non, émanant des établissements d'enseignement et de recherche français ou étrangers, des laboratoires publics ou privés. 


\title{
TRADR Project: Long-Term Human-Robot Teaming for Robot Assisted Disaster Response
}

\author{
Ivana Kruijff-Korbayová - Francis Colas • \\ Koen Hindriks • Mark Neerincx . \\ Petter Ögren · Mario Gianni · Tomáš \\ Svoboda - Rainer Worst
}

Received: date / Accepted: date

\begin{abstract}
This paper contains a description of the project TRADR: LongTerm Human-Robot Teaming for Robot Assisted Disaster Response. As robotic disaster relief systems are still scarce, any incident serious enough to render robot involvement will most likely involve a sequence of sorties over several hours, days and even months. TRADR focuses on the persistence of environment models, multi-robot action models, and human-robot teaming, in order to allow incremental capability improvement over the duration of a mission. TRADR applies a user centric design approach to disaster response robotics, with use cases involving the response to a medium to large scale industrial accident by teams consisting of human rescuers and several robots (both ground and airborne). The paper overviews the project objectives and motivation, the structure and approach, as well as the partners and related work.
\end{abstract}

Keywords disaster response robotics · persistent environment models . persistent multi-robot action models · persistent human-robot teaming . user-centric design

TRADR is an EU-funded Integrated Project in the FP7 Programme ICT: Cognitive Systems Interaction, Robotics (grant nr. 609763), running from November 2013 to December 2017. URL: www.tradr-project.eu

I. Kruijff-Korbayová (corresponding author)

Language Technology Lab, Deutsches Forschungszentrum für Künstliche Intelligenz (DFKI) Stuhlsatzenhausweg 3, 66123 Saarbrücken, Germany

Tel.: +49-681-85775.5356 Fax: +49-681-85775.5338, E-mail: ivana.kruijff@dfki.de

Francis Colas, Eidgenössische Technische Hochschule Zürich (ETH)

Koen Hindriks, Technische Universiteit Delft (TUD)

Mark Neerincx, Nederlandse Organisatie voor Toegepast Natuurwetenschappelijk Onderzoek (TNO)

Petter Ögren, Kungliga Tekniska Hoegskolan (KTH)

Mario Gianni, Università degli Studi di Roma "La Sapienza" (ROMA)

Tomáš Svoboda, České Vysoké Učení Technické v Praze (ČVUT)

Rainer Worst, Fraunhofer Institute for Intelligent Analysis and Information Systems 

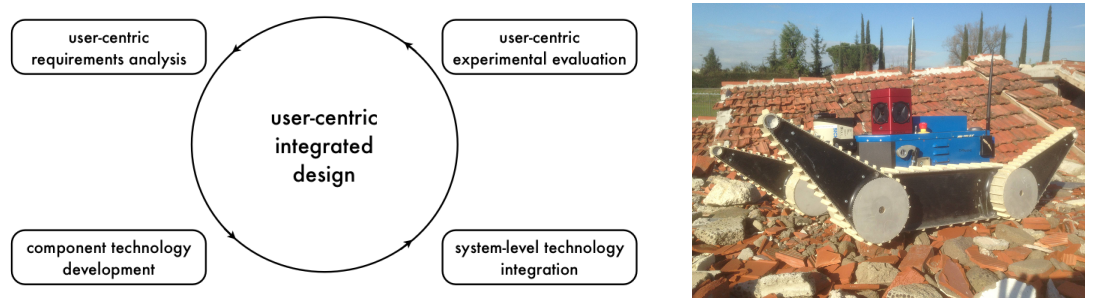

Fig. 1 TRADR one-year-round development cycle (1.), Absolem ground robot (r.).

\section{Introduction}

TRADR builds on the research and experience of the NIFTi project [30, 22]. Using a proven-in-practice user-centric design methodology (cf. Fig. 1), TRADR develops novel science and technology for human-robot teams to assist in disaster response efforts, over multiple sorties during a mission. TRADR aims to make experience persistent. The TRADR use cases involve response to a medium to large scale industrial accident by teams consisting of human rescuers and several ground and airborne robots, including the one in Fig. 1. The team collaborates to explore the environment and gather measurements and physical samples. TRADR enables the team to gradually develop its understanding of the disaster area over multiple, possibly asynchronous sorties (persistent environment models), to improve team members' understanding of how to work in the area (persistent multi-robot action models), and to improve team-work (persistent human-robot teaming). TRADR missions will ultimately stretch over several days in increasingly dynamic environments.

\subsection{Motivation}

A real disaster response takes longer than a single sortie into the area, just like life typically lasts longer than just one day. Deployments can last days, weeks, months, if not years, as witnessed recently in Japan (Fukushima) and in Northern Italy (Emiglia Romagna). Multiple robots need to be sent into the area, together (synchronous operation) or one after the other (asynchronous operations), to build up a better understanding of operating in the area. This understanding builds up gradually, and gets reflected in a developing (distributed) situation awareness [37], in how the team adapts to best coordinate its efforts (team-level), and how it learns to best execute its tasks (task-level).

Fig. 2 illustrates this premise. In May-June 2012, Northern Italy was hit by over 250 seismic events, causing widespread damage to an area rich in cultural heritage. Vigili del Fuoco, the Italian national rescue organisation responsible for disaster response, requested the NIFTi project $^{1}$ to assist in structure damage assessment. NIFTi fielded a human-robot team with a mobile

1 TRADR builds on the research and experience of the NIFTi project [30,22]. 


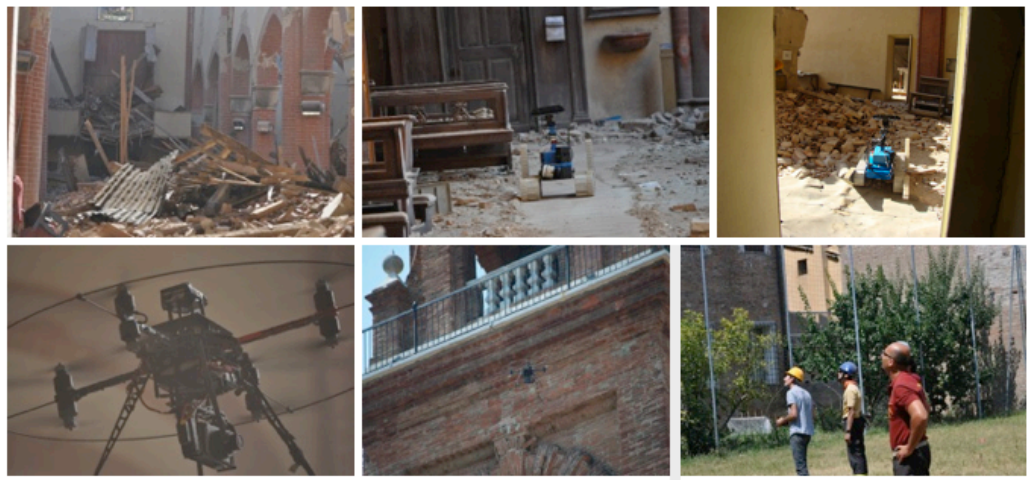

Fig. 2 Duomo in Mirandola, Northern Italy. Top: Main aisle (1.), UGV exploring eastern aisle (m.), top of the western aisle (r.). Bottom: UAV exploring eastern aisle (l.), exploring cracks in bell tower (m.), UAV team (r.).

command post, two unmanned ground vehicles (UGVs), and two quadcopter unmanned aerial vehicles (UAVs). The crucial insight from this deployment was the need for persistent, integrated situation awareness that builds up over multiple sorties during a mission, and that different kinds of robots each play complementary roles in this process [23].

\subsection{Project Partners}

The TRADR consortium consists of 12 partners, ${ }^{2}$ including 3 research institutes: DFKI (coordinator), Fraunhofer, TNO; 5 universities: ETH, KTH, ČVUT, ROMA and TUD; one industry partner: Ascending Technologies; and three end-user organizations, representatives of the fire-brigades from Germany (Stadt Dortmund Institut für Feuerwehr und Rettungstechnologie), Italy (Vigili del Fuoco directed by the Ministero Dell'interno) and the Netherlands (Gezamenlijke Brandweer). The partners contribute to the work packages listed in Sec. 3.2 according to their respective expertise. 8 of the partners have already collaborated very successfully in the NIFTi project.

\section{Related work}

Deployment of (teams of) UGVs and UAVs in various disaster response scenarios is the subject of several other European projects, e.g., ICARUS, DARIUS, SHERPA, TIRAMISU. But they do not address the persistence issues. The EU project STRANDS, on the other hand, aims at modeling the spatio-temporal dynamics in human 3D environments in order for a robot to adaptat to and exploit long-term experience in months-long autonomous operation. TRADR

\footnotetext{
2 Cf. the authors' list for full names of the institutes listed here only by an abbreviation. For more information on the partners, please visit the project website [41].
} 
deals with a human-robot team carrying out multiple sorties into an unstructured environment. Building on the experience of the consortium in the NIFTi project, TRADR uses key results from NIFTi as basis towards the development of persistent environment models, persistent multi-robot action models and persistent human-robot teaming.

Persistent multi-robot environment models are grounded in two different aspects: environment representation and adaptive action. So far, 3D mapping is essentially studied for a single robot starting from an empty map. We need to develop new data structures similar to octrees [44] and multi-resolution surfel maps [39] but with that added capabilities to integrate different sensor data from different robots, to scale to arbitrary environment sizes, and to cope with dynamic obstacles [35]. Impressive demonstrations of aggressive manoeuvres have shown the capabilities of UAVs but always in a closed environment with high-precision external tracking systems $[25,26]$. To replicate these results in field experiments, it is necessary to improve the performance of current state estimation techniques relying on vision or laser sensor to complement IMU measurements $[1,43]$. Contrary to UAVs where the difficulty lies often more in the control as they are unstable systems, UGVs research is more focused on path planning. A pletora of algorithms allow robots on flat ground to find optimal paths using robot constraints $[21,36]$ but few approaches investigate moving in a rough terrain by using flippers $[31,8]$ and these are not yet ready for large-scale or dynamic environments.

Persistent multi-robot action models Robots collaboration presupposes intention to collaborate, awareness of roles, partial knowledge, distinct beliefs, desires, capabilities and goals, whereas interaction would possibly be passive, unintentional or even forced [3]. These aspects have been applied in multi-robot localization [10], multi-robot exploration [20,7], multi-robot path planning [2], task allocation [13], collaborative dialogue [38], coalitions [42], negotiation [28], intention conflict resolution [18], team working [40], role allocation [29] and planning $[4,5,24,6]$. Although significant research results have been achieved in the last thirty years, a completely new concept of multi-robot collaboration comes up in TRADR, namely the concept of persistent collaboration. The great strength and novelty of the concept of persistent collaboration goes actually beyond time extension, as for example foreseen in the EU-projects ALIZ-E [11], or STRANDS [12]. The concept of persistence in multi-robot collaboration requires persistence to be verified through sorties where an enormous amount of data is collected by the robot team. The challenge in TRADR is to model how the information content of the data collected is preserved, and it is lifted to knowledge, while changing the team, changing the ways of communication and changing the experience gathered. Because this process is highly dynamic, we resort to the paradigm of world as memory [32] in which the gaps are hidden and compensated by inference. The great novelty is, therefore, in this capacity of inference to dissipate useless information and use the world as an outside memory, to keep only what is needed to grow experience. The 
difficulties here are given by the environment harshness and its compelling requests due to the mission goals. This, in turns, asks for strong communication structures at different layers, so as to assign at each sortie the correct role to the team members that intervene, if autonomously, by task inference if not by displaying the whole team current states and capacities to the command operators, in any case ensuring team dependability during plan execution. Persistence demands also consistent continuous information sharing which is especially hard in damaged environments and has never been experienced before. Moreover, it requires to pass from theories to operations and procedures that can last over time periods covering the mission duration. There exists so far a discrepancy between the multi-agent algorithms and their applicability to the multi-robot domains; persistent multi-robot collaboration has to bridge the gap both operatively and methodologically, as many of the existing formalizations are devoted to abstract agents with perfect communication and functioning. The framework that TRADR proposes for persistent multi-robot collaboration maintains a structure of memories, knowledge and environments, with different levels and mode of inferences [34], that can deal with learning paradigms to control and manage the generation of common, individual and collaborative goals. This model exploits the experience gained in NIFTi with working memories to manage the information flow from different components $[15,14,19]$.

Persistent human-robot teaming As robots become more sophisticated a tendency has arisen within HRI to perceive them as teammates rather than tools $[17,33]$; also in the context of USAR the importance of robots capable to operate as a (social) team-member has been acknowledged and addressed [9, 27]. Even though in NIFTi multiple robots were employed, they did not necessarily partake on the team-level; each robot was controlled by an individual operator taking orders from the human commander. Also in related projects like ICARUS and DARIUS, teams of heterogeneous robots are employed in a collaborative fashion, but they are controlled by human operators who provide the linkage between the robot teams and the rescue workers. The SHERPA project on the other hand has a stronger notion of human-robot collaboration, employing a metaphor of the human as "busy genius" who collaborates with a group of robots with different capabilities (the "SHERPA animals") towards a common (SAR) goal. In similar vein, within TRADR we want to go beyond an approach in which robots are mere tools under control of a human team, instead viewing the robots as team-members with a certain level of autonomy (e.g. semi-autonomous navigation, data gathering etc). To realize this, TRADR will develop a framework for coordination of human-robot teaming, which is build on agent-based technology [16]. This framework manages the different roles, objectives, responsibilities and expectation for members of the team (which consists of both robots and humans and which may change over different sorties) and allows for dynamical task-allocation depending on capabilities, capacities, task-load and chances of success. 

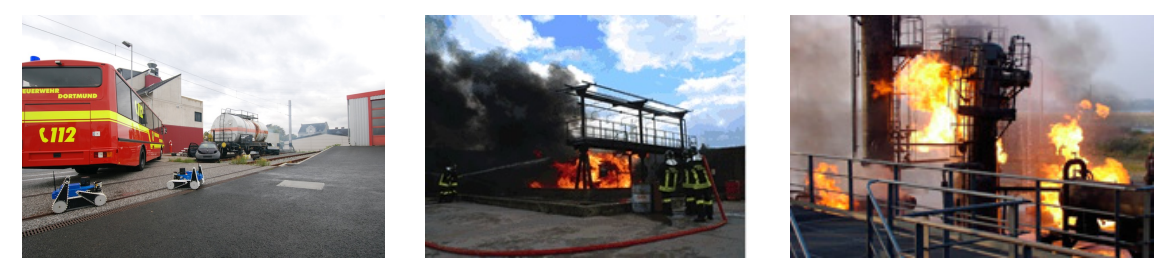

Fig. 3 Examples of training sites in Germany, Italy, and the Netherlands.

\section{Project Structure and Approach}

TRADR adopts a scenario-based roadmap, to drive iterative development and integration. The roadmap defines a single scenario setting, namely a largescale industrial disaster. Within this setting, the roadmap then defines yearly use cases which deal with situation assessment (e.g., through observation and sample gathering) under increasingly more complex circumstances. This is a kind of disaster where persistence is key to a successful mission. Missions take longer than a single sortie onto the scene, we need multiple robots to investigate the disaster from different angles (literally), and we need to use them over a number of sorties to gradually build up an assessment. The user organizations in the TRADR consortium provide training facilities to set up such a scenario. See Fig. 3 for examples.

\subsection{Year-By-Year Roadmap}

The goal in Yr1 is to enable a fixed human-robot team to gradually build up situation awareness of a static disaster site over multiple, asynchronous sorties.

In Yr2, TRADR moves to dealing with a dynamic environment. The goal is for a fixed team to build up situation awareness of a dynamic disaster site over multiple, synchronous or asynchronous sorties.

In Yr3, TRADR brings multiple robots into the field, during individual missions. This yields valuable insights in how environment models get fused, and may be used. TRADR builds on these insights to move task adaptation from a strictly individual focus, to a multi-robot setting: How could a robot learn from its use of information provided by others, to adapt its own tasks as well as anticipate requests for such collaboration in (future) plans?

In Yr4, multiple robots collaborate in various ways, including both synchronous and asynchronous collaboration. Persistence in modeling the environment covers an ever-increasing complexity in local and global dynamic events, appearing on an ever-larger spatiotemporal scale. Team competence gradually improves based on experience.

\subsection{Work Package Structure}

TRADR is structured into seven research\&development work packages: 
WP1: Persistent models for perception aims to provide sensory data from all involved robots registered in space and time, to keep creating and updating robot centric representations, and ground them into the world coordinate frame. The obtained representations are furnished to other WPs, which maintain higher level situation awareness.

WP2: Persistent models for acting addresses various levels of autonomy for the robotic platforms. It is anchored by the representations built in WP1 and enacts the collaborative plans of WP4.

WP3: Persistent models for distributed joint situation awareness has the aim to promote trustworthy and relevant tactical information about the physical environment, to provide a hierarchical representation of experiences which supports tactical decision making, and to support multi-modal interaction with the human team-members.

WP4: Persistent models for multi-robot collaboration deals with persistent collaboration among members of a robot team willing to act both together and individually. It develops a statistical-logical theory of flexible collaborative planning, and - based on this - a framework that exploits both logical and statistical inference from several knowledge levels and their partial integration. Implementation of the above framework requires to design algorithms that can cope with (i) team knowledge maintenance and updating, by knowledge and information sharing operations, and (ii) team-activity dynamic maintenance via the cycle of predict-what-needed and decide-to-collaborate.

WP5: Persistent models for human-robot teaming aims to to develop a logical-probabilistic framework for explaining: how a robot can determine interactive behavior for team-level coordination; how conflicts between actors can arise in the team, how these conflicts can be used to improve alignment between (robot's) private expectations and the other's behavior and how this can be reflected in an improved ability to determine interactive behavior.

WP6: System framework and integration specifies and sets up the TRADR technical system framework, develops adaptive control on the system level, and integrates WP components continuously into a single architecture.

WPr: User needs analysis and scenario-based evaluation performs a deep domain analysis with end-users, evaluates WP components on system-level, and performs end-user evaluations of the integrated systems.

\section{Conclusions}

We presented an overview of the TRADR project. TRADR advances the use of the user-centric methodology established in the NIFTi project, and builds on the experience and insights obtained through the deployment of the NIFTi system, that there is a need for persistent, integrated situation awareness gathered over multiple sorties during a mission, and that different kinds of robots each play complementary roles in this process. TRADR thus develops the capacity for persistent environment models, persistent multi-robot action models and persistent human-robot teaming. 


\section{References}

1. Achtelik, M., Bachrach, A., He, R., Prentice, S., Roy, N.: Stereo vision and laser odometry for autonomous helicopters in GPS-denied indoor environments. In: SPIE Unmanned Systems Technology XI (2009)

2. Bennewitz, M., Burgard, W., Thrun, S.: Optimizing schedules for prioritized path planning of multi-robot systems. In: ICRA, pp. 271-276 (2001)

3. Bratman, M.E.: Shared cooperative activity. Philosophical Review 101(2), 327-341 (1981)

4. Brenner, M.: Mapl: a framework for multiagent planning with partially ordered temporal plans. In: ICAPS (2003)

5. Brenner, M.: Continual collaborative planning for mixed-initiative action and interaction. In: AAMAS (3), pp. 1371-1374 (2008)

6. Brenner, M., Nebel, B.: Continual planning and acting in dynamic multiagent environments. Autonomous Agents and Multi-Agent Systems 19(3), 297-331 (2009)

7. Burgard, W., Moors, M., Stachniss, C., Schneider, F.E.: Coordinated multi-robot exploration. IEEE Transactions on Robotics 21(3), 376-386 (2005)

8. Colas, F., Mahesh, S., Pomerleau, F., Liu, M., Siegwart, R.: 3D path planning and execution for search and rescue ground robots. In: Intelligent Robots and Systems (IROS), 2013 IEEE/RSJ International Conference on. IEEE (2013)

9. Fincannon, T., Barnes, L.E., Murphy, R.R., Riddle, D.L.: Evidence of the need for social intelligence in rescue robots. In: IEEE/RSJ International Conference on Intelligent Robots and Systems (IROS), vol. 2, pp. 1089-1095. IEEE (2004)

10. Fox, D., Burgard, W., Kruppa, H., Thrun, S.: Collaborative multi-robot localization. In: DAGM-Symposium, pp. 15-26 (1999)

11. FP7-ICT-2009-4, E.I.C.: Aliz-e, adaptive strategies for sustainable long-term social interaction, id 248116. URL http://www.aliz-e.org/

12. FP7-ICT-2012-9, E.I.C.: Strands: Spatio-temporal representations and activities for cognitive control in long-term scenarios. URL http://www.cs.bhm.ac.uk/ nah/

13. Gerkey, B.P., Mataric, M.J.: Murdoch: Publish/subscribe task allocation for heterogeneous agents. In: AAAI/IAAI, p. 1070 (2000)

14. Gianni, M., Papadakis, P., Pirri, F.: Awareness in mixed initiative planning. In: Proceedings of the AAAI Fall Symposium Series (2011)

15. Gianni, M., Papadakis, P., Pirri, F., Liu, M., Pomerleau, F., Colas, F., Zimmerman, K., Svoboda, T., Petricek, T., Kruijff, G.J.M., Zender, H., Khambhaita: A unified framework for planning and execution-monitoring of mobile robots. In: Proceedings of the AAAI-11 Workshop on Automated Action Planning for Autonomous mobile Robots (2011)

16. Hindriks, K.V.: Programming rational agents in goal. Multi-Agent Programming: Languages, Tools and Applications pp. 119-157 (2009)

17. Hoffman, G., Breazeal, C.: Collaboration in human-robot teams. In: Proc. of the AIAA 1st Intelligent Systems Technical Conference, Chicago, IL, USA (2004)

18. Jung, H., Tambe, M., Zhang, W., Shen, W.M.: Towards large-scale conflict resolution: initial results. In: MultiAgent Systems, 2000. Proceedings. Fourth International Conference on, pp. $403-404$ (2000)

19. Khambhaita, H., Kruijff, G., Mancas, M., Gianni, M., Papadakis, P., Pirri, F., Pizzoli, M.: Help me to help you: how to learn intentions, actions and plans. In: AAAI 2011 Spring Symposium "Help Me Help You: Bridging the Gaps in Human-Agent Collaboration" (2011)

20. Ko, J., Stewart, B., Fox, D., Konolige, K., Limketkai, B.: A practical, decision-theoretic approach to multi-robot mapping and exploration. In: Intelligent Robots and Systems, 2003. (IROS 2003). Proceedings. 2003 IEEE/RSJ International Conference on, vol. 4 , pp. $3232-3238$ vol.3 (2003)

21. Koenig, S., Likhachev, M.: Fast replanning for navigation in unknown terrain. IEEE Transactions on Robotics 16(3), 354-363 (2005)

22. Kruijff, G., Colas, F., Svoboda, T., van Diggelen, J., Balmer, P., Pirri, F., Worst, R.: Designing intelligent robots for human-robot teaming in urban search \& rescue. In: Proceedings of the AAAI 2012 Spring Symposium on Designing Intelligent Robots (2012) 
23. Kruijff, G., Tretyakov, V., Linder, T., Pirri, F., Gianni, M., Papadakis, P., Pizzoli, M., Sinha, A., E, P., Corrao, S., Priori, F., Febrini, S., Angeletti, S.: Rescue robots at earthquake-hit Mirandola, Italy. In: Proc. of the 10th IEEE International Symposium on Safety, Security, and Rescue Robotics (SSRR-2012). IEEE Press, College Station, TX, USA (2012)

24. Kruijff, G.J.M., Brenner, M., Hawes, N.: Continual planning for cross-modal situated clarification in human-robot interaction. In: RO-MAN, pp. 592-597 (2008)

25. Lupashin, S., Schoellig, A., Sherback, M., D'Andrea, R.: A simple learning strategy for high-speed quadrocopter multi-flips. In: IEEE International Conference on Robotics and Automation (ICRA) (2010)

26. Michael, N., Fink, J., Kumar, V.: Cooperative manipulation and transportation with aerial robots. Autonomous Robots (2010)

27. Murphy, R.R.: Human-robot interaction in rescue robotics. Systems, Man, and Cybernetics, Part C: Applications and Reviews, IEEE Transactions on 34(2), 138-153 (2004)

28. Murray, C., Gordon, G.: Multi-robot negotiation: Approximating the set of subgame perfect equilibria in (2006)

29. Nair, R., Tambe, M., Marsella, S.: Role allocation and reallocation in multiagent teams: towards a practical analysis. In: Proceedings of the second international joint conference on Autonomous agents and multiagent systems, AAMAS '03, pp. 552-559. ACM, New York, NY, USA (2003)

30. NIFTi (2013). URL http://www.nifti.eu

31. Okada, Y., Nagatani, K., Yoshida, K., Tadokoro, S., Yoshida, T., Koyanagi, E.: Shared autonomy system for tracked vehicles on rough terrain based on continuous threedimensional terrain scanning. Journal of Field Robotics 26(6), 875-893 (2011)

32. O'Regan, K.J.: Solving the "real" mysteries of visual perception: The world as an outside memory. Robotics, IEEE Transactions on 46(3), 461 -488 (1992)

33. Ososky, S., Schuster, D., Jentsch, F., Fiore, S., Shumaker, R., Lebiere, C., Kurup, U., Oh J., Stentz, A.: The importance of shared mental models and shared situation awareness for transforming robots from tools to teammates. In: SPIE Defense, Security, and Sensing, pp. 838,710-838,710. International Society for Optics and Photonics (2012)

34. Pirri, F.: The well-designed logical robot: Learning and experience from observations to the situation calculus. Artif. Intell. 175(1), 378-415 (2011)

35. Pomerleau, F., Krüsi, P., Colas, F., Furgale, P., Siegwart, R.: Long-term 3D map maintenance in dynamic environments. In: Robotics and Automation (ICRA), 2014 IEEE International Conference on. IEEE (2014)

36. Rufli, M., Ferguson, D., Siegwart, R.: Smooth path planning in constrained environments. In: IEEE International Conference on Robotics and Automation (ICRA) (2009)

37. Salmon, P., Stanton, N., Walker, G., Jenkins, D.: Distributed Situation Awareness: Theory, Measurement, and Application to Teamwork. Human Factors in Defence. Ashgate (2009)

38. Sidner, C.L.: Collaborative agents for 2 d interfaces and 3 d robots. In: AH, p. 2 (2004)

39. Stueckler, J., Behnke, S.: Multi-resolution surfel maps for efficient dense 3D modeling and tracking. J. of Visual Communication and Image Representation (2013). To appear

40. Tambe, M.: Towards flexible teamwork. J. Artif. Int. Res. 7(1), 83-124 (1997)

41. TRADR (2014). URL http://www.tradr-project.eu

42. Vig, L., Adams, J.A.: A framework for multi-robot coalition formation. In: IICAI, pp. 347-363 (2005)

43. Weiss, S., Achtelik, M.W., Lynen, S., Chli, M., Siegwart, R.: Real-time onboard visualinertial state estimation and self-calibration of MAVs in unknown environments. In: IEEE International Conference on Robotics and Automation (ICRA) (2012)

44. Wurm, K., Hornung, A., Bennewitz, M., Stachniss, C., Burgard, W.: Octomap: A probabilistic, flexible, and compact 3d map representation for robotic systems. In: Proc. of the ICRA 2010 workshop on best practice in 3D perception and modeling for mobile manipulation, vol. 2 (2010) 


\section{Authors' Profiles}
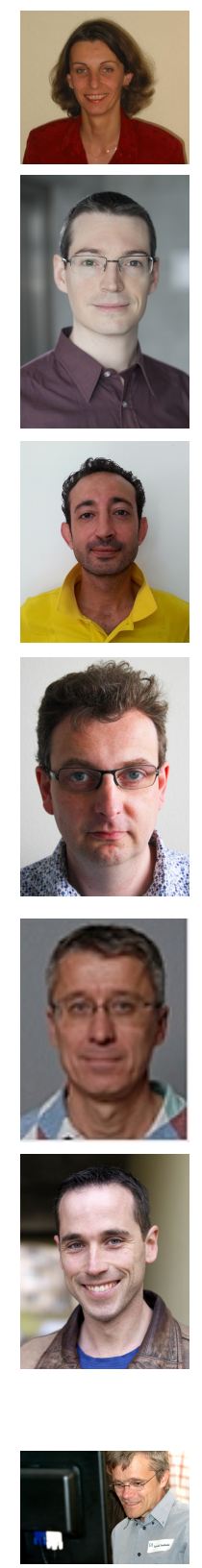

Koen Hindriks: MSc degree from the University of Groningen; PhD degree from Utrecht University; part of $\mathrm{PhD}$ candidature at the University of Toronto. Currently Assistant Professor at the Delft University of Technology, Department of Electrical Engineering, Mathematics and Computer Science. Research in multi-agent technology.

Mark Neerincx After M.Sc. he worked at the TNO Institute of Preventive Health Care, the University of Leiden and the University of Amsterdam. After Ph.D. he became research scientist at TNO Human Factors and, from 2000, headed the Usability Engineering group. He is also (part-time) full professor in Human-Centred Computing at Delft University of Technology. He has extensive experience in applied and fundamental research and conducted empirical studies in diverse domains, such as process control, defence, aerospace, health, public safety and e-commerce. Petter Ögren: MSc and PhD degree from KTH - Royal Institute of Technology, Sweden; part of $\mathrm{PhD}$ candidature at Princeton University. Currently Associate Professor at KTH, Department of Computer Vision and Active Perception (CVAP), School of Computer Science and Communication. Research interests include multi-agent coordination, formations, obstacle avoidance, intelligent teleoperation and applying ideas from computer games to robot control problems.

Tomáš Svoboda: Ph.D. in artificial intelligence and biocybernetics from the Czech Technical University in Prague in 2000. Post-doc with the Computer Vision Group at the ETH Zurich (Swiss Federal Institute of Technology) for 3 years. Currently assistant professor and deputy head of the department of Cybernetics at the Czech Technical University. Research interests include multicamera systems for scene understanding and telepresence, tracking and motion analysis.

Dipl.-Inform. Rainer Worst: Senior researcher at Fraunhofer IAIS. Diploma in Computer Science (Univ. Bonn, 1981). IT-Expert at a consulting company in Wiesbaden. In 1984 joined the former GMD (National Research Center for Information Technology) in Sankt Augustin, which merged 1999 with Fraunhofer. Experience as a consultant, researcher and project leader in the areas Software Engineering, Quality Management, Autonomous Systems, and Virtual Environments. 